\title{
Eosinophilic Granulomatosis with Polyangiitis (Churg-Strauss Syndrome) Complicated by Perforation of the Small Intestine and Cholecystitis
}

\author{
Yoichi Ohnuki ${ }^{1}$, Yusuke Moriya ${ }^{1}$, Sachiko Yutani ${ }^{1}$, Atsushi Mizuma ${ }^{1}$, Taira Nakayama ${ }^{1}$, \\ Yuko Ohnuki ${ }^{2}$, Shuji $\mathrm{Uda}^{3}$, Chie Inomoto ${ }^{4}$, Soichiro Yamamoto ${ }^{3}$, \\ Naoya Nakamura ${ }^{4}$ and Shunya Takizawa ${ }^{1}$
}

\begin{abstract}
:
We report a case of eosinophilic granulomatosis with polyangiitis (EGPA; formerly known as ChurgStrauss syndrome) complicated by perforation of the small intestine and necrotizing cholecystitis. A 69-yearold man with a history of bronchial asthma was admitted with mononeuritis multiplex. The laboratory findings included remarkable eosinophilia. He was treated with corticosteroids and his laboratory indices showed improvement; however, his functional deficits remained. His neuropathy gradually improved after the addition of intravenous immunoglobulin (IVIG). He was subsequently treated with oral prednisolone (40 $\mathrm{mg} /$ day) as maintenance therapy. Within a month after finishing IVIG, he developed perforation of the small intestine and necrotizing cholecystitis. Intestinal perforation has often been reported as a gastrointestinal complication of EGPA. In contrast, cholecystitis is a rare complication. We report this case because the manifestation of more than one complication is extremely rare. Gastrointestinal symptoms may be a complication of EGPA itself and/or immunosuppressive treatment.
\end{abstract}

Key words: Churg-Strauss syndrome, eosinophilic granulomatosis with polyangiitis, perforation of small intestine, cholecystitis

(Intern Med 57: 737-740, 2018)

(DOI: 10.2169/internalmedicine.8975-17)

\section{Introduction}

The term eosinophilic granulomatosis with polyangiitis (EGPA) was recommended, in the 2012 revised nomenclature for vasculitides (1), as the preferred term for ChurgStrauss syndrome. EGPA is an uncommon disease characterized by asthma, hypereosinophilia, necrotizing vasculitis and extravascular granulomas (2). It sometimes involves the heart, lungs, gastrointestinal tract, kidneys or central nervous system (3). Corticosteroids are the cornerstone of treatment for EGPA. A French vasculitis study group reported that EGPA with or without poor prognostic factors can be effec- tively treated with first-line corticosteroids and immunosuppressive therapy $(4,5)$. In patients who fail to respond to corticosteroids and immunosuppression, high-dose intravenous immunoglobulin (IVIG) is recommended (3). We herein report a case of EGPA with severe neuropathy in a patient who was treated with steroids and IVIG and who subsequently developed perforation of the small intestine and cholecystitis as complications.

\section{Case Report}

A 69-year-old man with a history of bronchial asthma experienced hypoesthesia and muscle weakness, initially in the

${ }^{1}$ Division of Neurology, Department of Internal Medicine, Tokai University School of Medicine, Japan, ${ }^{2}$ Department of Molecular Life Science Basic Medical Science and Molecular Medicine, Tokai University School of Medicine, Japan, ${ }^{3}$ Department of Gastroenterological Surgery, Tokai University School of Medicine, Japan and ${ }^{4}$ Department of Pathology, Tokai University School of Medicine, Japan Received: February 1, 2017; Accepted: June 18, 2017; Advance Publication by J-STAGE: December 21, 2017 Correspondence to Dr. Yoichi Ohnuki, yohnuki@is.icc.u-tokai.ac.jp 


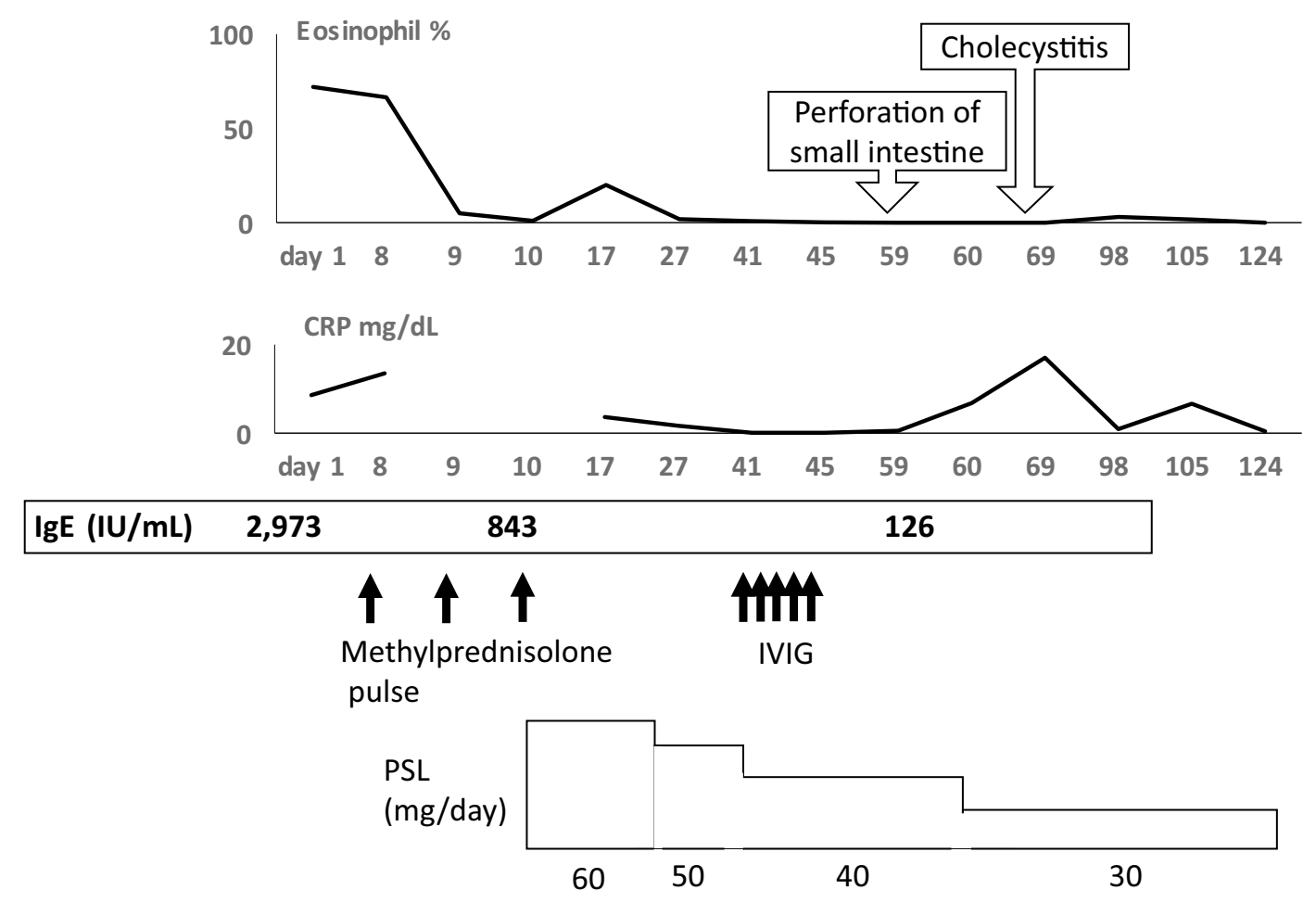

Figure 1. The laboratory data over the clinical course: eosinophil, C-reactive protein (CRP), immunoglobulin E (IgE). Methylprednisolone (1 g/day) was administered for 3 days. Immunoglobulin $(400 \mathrm{mg} / \mathrm{kg} / \mathrm{day})$ was administered for 5 days. Perforation of the small intestine occurred on day 59 after admission. Cholecystitis occurred on day 68 after admission.

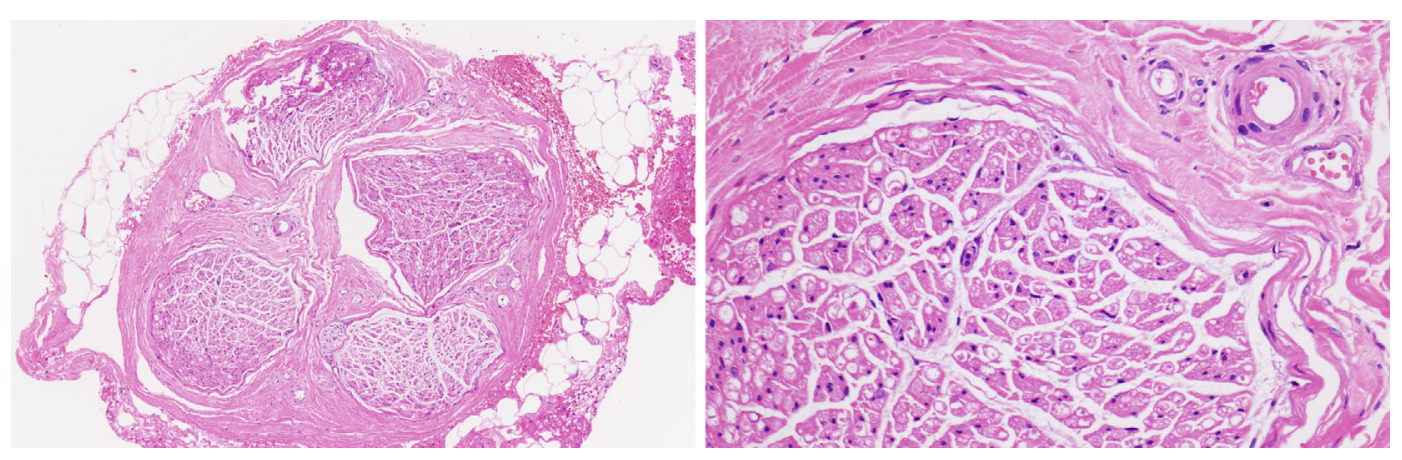

Figure 2. The histopathological examination of a sural nerve biopsy specimen. Apparent inflammatory cell infiltration and nerve fiber loss were not observed.

left lower limb, and then in all 4 limbs within 2 weeks. He was admitted to our hospital at 6 weeks after the onset of symptoms. His temperature was $36.9^{\circ} \mathrm{C}$, his pulse 108 beats/ min and regular, and his blood pressure was 158/92 $\mathrm{mmHg}$. Chest auscultation revealed no rales at the bilateral chest, and no abdominal abnormalities were found on a physical examination. Neurological findings indicated muscle weakness in bilateral wrist extension, wrist flexion, and in the quadriceps, hamstrings, and tibialis anterior muscles (manual muscle test score: 1-2). He also had hypoesthesia and disturbance of the positional sense of the sole of the left foot. A neurological examination indicated mononeuritis multiplex. Laboratory studies showed the following values: white blood cell count, $4,6200 / \mu \mathrm{L}$ with $72.0 \%$ eosinophils; C-reactive protein, $8.59 \mathrm{mg} / \mathrm{dL}$; IgE, $2973 \mathrm{IU} / \mathrm{mL}$; and erythrocyte sedimentation rate (ESR), $43 \mathrm{~mm} / \mathrm{h}$ (Fig. 1). The patient was negative for antinuclear antibodies, myeloperoxidase-specific antineutrophil cytoplasmic antibodies (MPO-ANCA), and proteinase-3 specific antineutrophil cytoplasmic antibodies (PR3-ANCA). His rheumatoid factor level was $107 \mathrm{IU} / \mathrm{mL}$. With a history of bronchial asthma, eosinophilia, and mononeuritis multiplex, he met three of the six American College of Rheumatology (ACR) criteria for Churg-Strauss syndrome. However, sural nerve biopsy did not reveal eosinophilic infiltration, vasculitis or the loss of nerve fibers (Fig. 2), and he did not qualify for positive diagnosis according to the ACR criteria, which require four or more of the six items (6). He was diagnosed with definite EGPA 
a

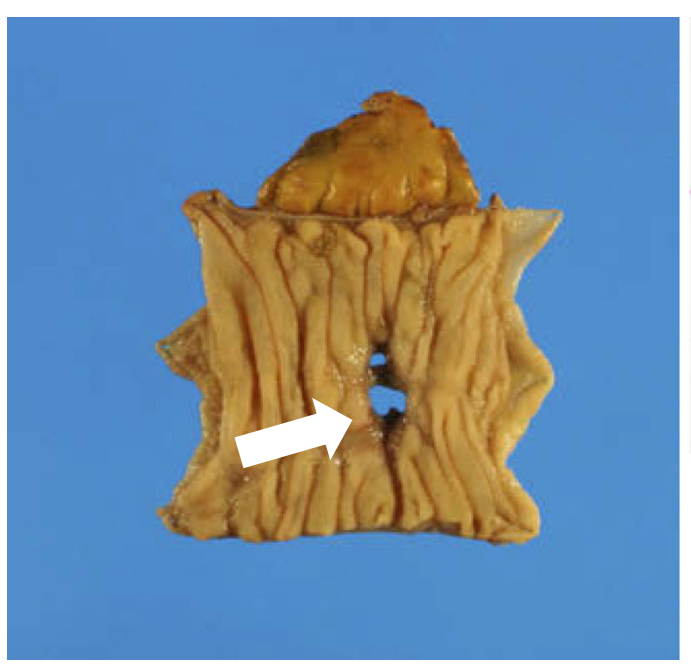

b

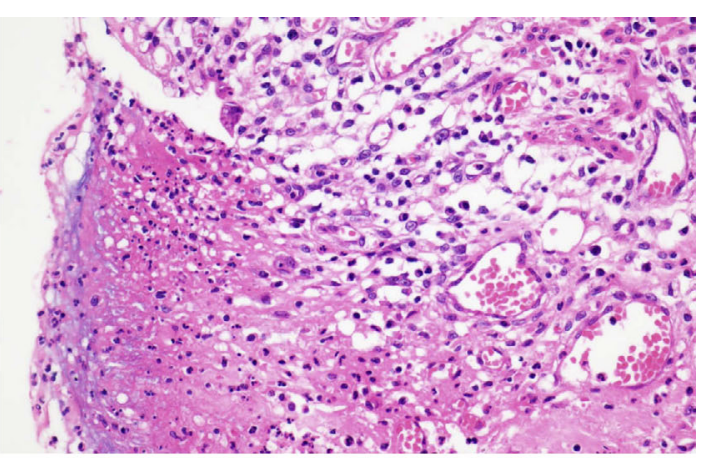

Figure 3. Partial resection of the small intestine. (a) Perforation was identified in the resected specimen (arrow). (b) The histopathological findings of the resected intestine. Neutrophils and plasma cells were observed. We did not detect eosinophilic infiltration.

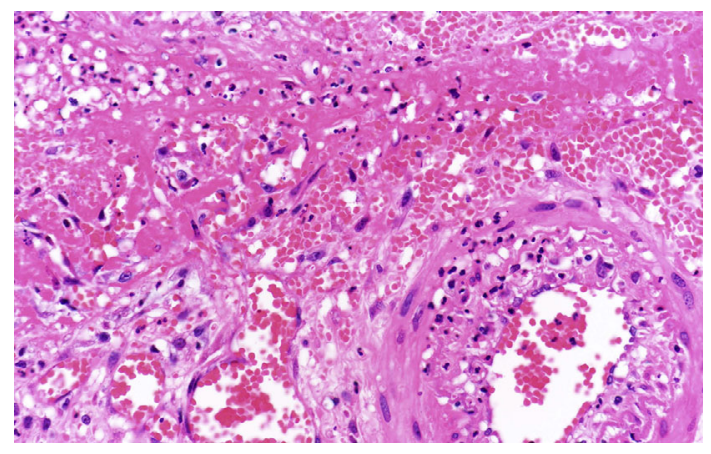

Figure 4. The histological examination of the gallbladder, and cholecystectomy. The histological examination of the gallbladder revealed neutrophilic infiltration (leukocytoclasia) and vasculitis.

based on the criteria of the Ministry of Health, Labor and Welfare in Japan (1998). Initial methylprednisolone pulse treatment (1 g/day for 3 days) and oral prednisolone (60 $\mathrm{mg} /$ day for 2 weeks) reduced his peripheral eosinophils to $1.9 \%$ (eosinophil counts; $167 / \mu \mathrm{L}$ ), his CRP level to 1.65 $\mathrm{mg} / \mathrm{dL}$, and his ESR to $16 \mathrm{~mm} / \mathrm{h}$; however, his muscle weakness did not improve. We did not give him additional immunosuppressants because he developed a severe urinary tract infection after the methylprednisolone treatment. Additional treatment with IVIG $400 \mathrm{mg} / \mathrm{kg} /$ day for 5 days tended to improve his hypoesthesia and muscle weakness. He continued to take oral prednisolone (40 mg/day) (Fig. 1). Fourteen days after the IVIG course, abdominal pain suddenly developed with severe distention and muscular defense over the entire abdomen. Laboratory studies showed the following values: white blood cell count, $19,000 / \mu \mathrm{L}$ with $0.0 \%$ eosinophils; and C-reactive protein, $0.52 \mathrm{mg} / \mathrm{dL}$. An abdominal X-ray and computerized tomography showed free air in the abdomen, suggesting the perforation of the gastrointesti- nal tract, and a part of the small intestine was resected. A histopathological examination revealed inflammation, but not angiitis, eosinophilic infiltration or granuloma (Fig. 3). On the 8th postoperative day, he had a fever of $38^{\circ} \mathrm{C}$. Abdominal CT showed that his gallbladder was thickened and he was diagnosed with necrotizing cholecystitis. Cholecystectomy was performed on the same day. The histopathological findings showed necrosis and vasculitis, but not eosinophilic infiltration or granulomatous inflammation (Fig. 4). On manual muscle testing, his muscle strength gradually improved to 3-4. He subsequently received oral prednisolone (30 mg/day) as maintenance therapy. There was no recurrence of fever or abdominal pain. He was transferred to a rehabilitation hospital at 5 months after admission.

\section{Discussion}

Corticosteroids are first-line therapy for EGPA in patients without severe organ involvement (3), and can be slowly tapered after a clinical response is achieved, usually within a few weeks (7). Koike et al. found that IVIG was effective for improving the residual neuropathy of patients with EGPA during disease remission, as indicated by their laboratory indices, after initial immunosuppressive treatment in their multicenter, double-blind, 3-arm treatment period, randomized, pre-post trial (8). In accordance with their report, the residual neuropathy of our patient improved after IVIG treatment.

Our case did not show EGPA-specific histopathological findings, such as eosinophilic infiltration or granuloma formation. However, because of the widespread use of glucocorticoid therapy and/or the small number of cases in which biopsy specimens are available for examination, it is where for granulomatosis to be observed in pathological examinations (3). 
Gastrointestinal involvement often occurs due to the eosinophilic infiltration of the gastrointestinal mucosa and more frequently affects the small intestine (3). Ulceration, perforation and stenosis of the gastrointestinal tract are thought to be the results of ischemia due to vasculitis (9). Moreover, acute ulcers or the perforation of the gastrointestinal tract often occur in association with steroid treatment (9). On the other hand, cholecystitis is rare as a complication of EGPA. A search of the PubMed database found 12 previous case reports of EGPA patients with cholecystitis, including one with erosion of the large intestine and cholecystitis (10). We could not find any EGPA cases with perforation of the small intestine or cholecystitis.

Steroid therapy is often used to treat EGPA, but steroids can enhance the fibrotic changes of the intimal tissue, which could cause intestinal perforation due to vascular obstruction (11). The prolonged use of steroids may cause gastrointestinal involvement, and switching to alternative therapies should be considered. Our patient received IVIG therapy as an additional treatment, but also continued to take oral prednisolone. We might have to reconsider our treatment regime. However, it is difficult to distinguish clinically whether the gastrointestinal involvements, including intestinal perforation and cholecystitis, occurred due to vasculitis or immunosuppression. Meticulous care must be taken when a patient receives long-term steroid treatment.

\section{Conclusion}

We herein reported a case of EGPA that was complicated by both perforation of the small intestine and necrotizing cholecystitis after the treatment of neuropathy with steroids and IVIG.

The authors state that they have no Conflict of Interest (COI).

\section{References}

1. Jennette JC, Falk RJ, Bacon PA, et al. 2012 revised International Chapel Hill Consensus Conference Nomenclature of Vasculitides. Arthritis Rheum 65: 1-11, 2013.

2. Churg J, Strauss L. Allergic granulomatosis, allergic angiitis, and periarteritis nodosa. Am J Pathol 27: 277-301, 1951.

3. Greco A, Rizzo MI, Cirdilio AD, et al. Churg-Strauss syndrome. Autoimmun Rev 14: 341-348, 2015.

4. Cohen P, Pagnoux C, Mahr A, et al. Churg-Strauss syndrome with poor-prognosis factors: a prospective multicenter trial comparing glucocorticoids and six or twelve cyclophosphamide pulses in forty-eight patients. Arthritis Rheum 57: 686-693, 2007.

5. Ribi C, Cohen P, Pagnoux C, et al. Treatment of Churg-Strauss syndrome without poor-prognosis factors. Arthritis Rheum 58: 586-594, 2008.

6. Mase AT, Hunder GG, Lie JT, et al. The American College of Rheumatology 1990 Criteria for the Classification of ChurgStrauss Syndrome. Arthritis Rheum 33: 1094-1100, 1990.

7. Shinico RA, Bottero P. Churg-Strauss angiitis. Best Pract Res Clin Rheumatol 23: 355-366, 1990.

8. Koike H, Akiyama K, Saito T, Sobue G. The Research Group for IVIg for EGPA/CSS in Japan. Intravenous immunoglobulin for chronic residual peripheral neuropathy in eosinophilic granulomatosis with polyangiitis (Churg-Strauss syndrome): a multicenter, double-blind trial. J Neurol 262: 752-759, 2015.

9. Murakami S, Misumi M, Sakata H, et al. Churg-Strauss syndrome manifesting as perforation of the small intestine: report of a case. Surg Today 34: 788-792, 2004.

10. Suzuki M, Nabeshima K, Miyazaki M, Yoshimura H, Tagawa S, Shiraki K. Churg-Strauss syndrome complicated by colon erosion, acalculous cholecystitis and liver abscesses. World J Gastroenterol 11: 5248-5250, 2005.

11. Venditti D, Valerio B, Ielpo B, Buonomo O, Petrella G. Bowel perforations in a patient affected by Churg-Strauss syndrome under high-dose steroid treatment: will alternative drugs reduce risk of surgery? Rheumatol Int 31: 1239-1241, 2011.

The Internal Medicine is an Open Access article distributed under the Creative Commons Attribution-NonCommercial-NoDerivatives 4.0 International License. To view the details of this license, please visit (https://creativecommons.org/licenses/ by-nc-nd/4.0/).

(C) 2018 The Japanese Society of Internal Medicine Intern Med 57: 737-740, 2018 